\title{
The Kernel of the Stochastic Cooperative Game
}

\author{
Hongxin Bai \\ Mathematics and Computer Department, Baoding University, Baoding 071000, China \\ E-mail: baihongxin1@126.com
}

Shang Li

Baoding Municipal Experimental Primary School, Baoding 071000, China

E-mail: 1ssy117@126.com

Zhenzhen $\mathrm{He}$

Management Department, Baoding University, Baoding 071000, China

E-mail: hellozhenzhen@126.com

\begin{abstract}
Based on the concept of the stochastic cooperative game, the concepts such as the kernel and the nucleolus of the countermeasure are extended in this article, and many concepts such as the maximum excess value, the kernel and the nucleolus of the stochastic cooperative countermeasure are defined, and some characters and properties, and the relationships among the kernel, the nucleolus and the minimum core of the stochastic cooperative game are discussed.
\end{abstract}

Keywords: Minimum core, Nucleolus, Kernel

\section{Basic concepts and marks}

The stochastic cooperative game introduced by Suijs et al (1995) is denoted by $\Gamma=\left(N,\left\{\mathrm{X}_{S}\right\}_{S \subset N},\left(\succ_{i}\right)_{i \in \mathrm{N}}\right)$, and $\mathrm{N}$ is the set of player, and $\mathrm{X}_{S}$ is the payment function of the alliance $\mathrm{S}$, and each stochastic payment has limited anticipation, and $\left(\succ_{i}\right)_{i \in \mathrm{N}}$ is the optimal relation about the player $i$.

The stochastic payment of the alliance $\mathrm{S}, \mathrm{X}_{S}$, is denoted by a binary pair $\left(d^{S}, r^{S}\right) \in R^{S} \times R^{S}$, and it satisfies $\sum_{i \in S} d_{i} \leq 0, \sum_{i \in S} r_{i}=1, r_{i} \geq 0$, and $i \in S$. According to $\left(d^{S}, r^{S}\right)$, the stochastic payment equals to $d_{i}+r_{i} X_{S}$, and is denoted by $\left(d^{S}, r^{S}\right)_{i} \cdot\left(d^{S}, r^{S}\right)_{i}$ is a stochastic variable, and the set of all payments of the alliance $\mathrm{S}$ is denoted by $Z(S)$. The set of all individual reasonable payments of the alliance $\mathrm{S}$ is denoted by $\operatorname{IR}(S)$, i.e.

$\operatorname{IR}(S)=\left\{\left(d^{S}, r^{S}\right) \in Z(S) \mid \forall i \in S: d_{i}+r_{i} X_{S} \succ_{i} X_{\{i\}}\right\}$

Suppose $\left(d^{N}, r^{N}\right),\left(\tilde{d}^{N}, \tilde{r}^{N}\right) \in Z(N)$ are two stochastic payments, and if a alliance S exists, for all $i \in S$, $\tilde{d}_{i}+\tilde{r}_{i} X_{S} \succ_{i} d_{i}+r_{i} X_{S}$, and $\sum_{i \in S}\left(d^{N}, r^{N}\right)_{i} \leq X_{S}$, so $\left(\tilde{d}^{N}, \tilde{r}^{N}\right)$ is the optimal one in $\left(d^{N}, r^{N}\right)$ about $\mathrm{S}$, i.e. $\left(\tilde{d}^{N}, \tilde{r}^{N}\right) \succ_{S}\left(d^{N}, r^{N}\right)$.

Define the set of all un-optimal payments of the stochastic cooperative game $\Gamma$ as the core of $\Gamma$, and it is denoted by $\operatorname{core}(\Gamma)$, i.e. 
$\operatorname{core}(\Gamma)=\left\{\left(d^{N}, r^{N}\right) \mid\left(d^{N}, r^{N}\right) \in I R(N),\right\}=\left\{\quad\left(d^{N}, r^{N}\right) \quad \mid\left(d^{N}, r^{N}\right) \in I R(N), \quad\right.$ and $\quad \mathrm{S} \quad$ and $\left(\tilde{d}^{N}, \tilde{r}^{N}\right) \in Z(N)$ don't exist to make $\left(\tilde{d}^{N}, \tilde{r}^{N}\right) \succ_{S}\left(d^{N}, r^{N}\right)$.

\section{Kernel of the stochastic cooperative game}

Definition 2.1: Suppose $\Gamma=\left(N,\left\{X_{S}\right\}_{S \subset N},\left(\succ_{i}\right)_{i \in N}\right)$ is the stochastic cooperative game $\forall S \subseteq N$ with n players, $\left(d^{N}, r^{N}\right) \in Z(N), \forall(\tilde{d}, \tilde{r}) \in Z(S)$, so call $e[S,(d, r)]=\sum_{i \in S}\left(\tilde{d}^{S}, \tilde{r}^{S}\right)_{i}-\sum_{i \in S}\left(d^{N}, r^{N}\right)_{i}$ is the excess value of the alliance $\mathrm{S}$ about $\left(d^{N}, r^{N}\right)$.

This value reflects the attitude of the alliance $\mathrm{S}$ to the distribution $\left(d^{N}, r^{N}\right) . e[S,(d, r)]$ is bigger, $\left(d^{N}, r^{N}\right)$ is not welcomed by S. For the fixed $\left(d^{N}, r^{N}\right) \in Z(N)$, the excess value of $2^{n}$ subsets $S \subseteq N$ of $\mathrm{N}$ about $\left(d^{N}, r^{N}\right)$. And rank them from big to small, a $2^{n}$ dimensional vector can be obtained.

$Q(d, r)=\left(Q_{1}(d, r), Q_{2}(d, r), \cdots, Q_{2^{n}}(d, r)\right)$

Where, $Q_{i}(d, r)=e\left[S_{i},(d, r)\right] \quad\left(i=1,2, \cdots, 2^{n}\right), S_{1}, S_{2}, \cdots, S_{2^{n}}$ is an array of all subsets of $\mathrm{N}$ (about $\left.\left(d^{N}, r^{N}\right)\right)$ and satisfies

$e\left[S_{1},(d, r)\right] \geq e\left[S_{2},(d, r)\right] \geq \cdots \geq e\left[S_{2^{n}},(d, r)\right]$

$\leq_{L}$ or $\geq_{L}$ denotes the dictionary sequence in the nucleolus.

Definition 2.2: Suppose $\Gamma=\left(N,\left\{X_{S}\right\}_{S \subset N},\left(\succ_{i}\right)_{i \in N}\right)$ is the stochastic cooperative game with $\mathrm{n}$ players, $\varepsilon \in R, C_{\varepsilon}(\Gamma)=\left\{\left(d^{N}, r^{N}\right) \in Z(N) \mid e[S,(d, r)] \leq \varepsilon, S \subset N, S \neq \varnothing, N\right\}$, so $C_{\varepsilon}(\Gamma)$ is the strong $\varepsilon$ core of $C_{\varepsilon}(\Gamma)$. When $\varepsilon=0, \varepsilon=0$, i.e. the core $C(\Gamma)$ of $\Gamma$ is the 0 nucleolus, and when $\varepsilon$ is small enough, $C_{\varepsilon}(\Gamma)=\phi$; and when $\varepsilon$ is big enough, $C_{\varepsilon}(\Gamma) \neq \phi$. Suppose $\varepsilon_{0}=\inf \left\{\varepsilon \mid C_{\varepsilon}(\Gamma) \neq \phi\right\}$, so $C_{\varepsilon}(\Gamma)$ is the minimum core of $\Gamma$, i.e. $L C(\Gamma)$.

Note $\mathrm{M}_{i j}$ is the alliance of the alliances containing i not $\mathrm{j}$ in the stochastic cooperative game $\Gamma$, i.e.

$\mathrm{M}_{i j}=\left\{S \subset 2^{n} \mid i \in S, j \notin S\right\}$

Definition 2.3: For $\forall\left(d^{N}, r^{N}\right) \in Z(N)$,

$s_{i j}=\max _{S \in \mathrm{M}_{i j}} e[S,(d, r)]$

is the maximum excess value of the player i exceeding $\mathrm{j}$ at the position of $\left(d^{N}, r^{N}\right)$.

Definition 2.4: Suppose $\Gamma=\left(N,\left\{X_{S}\right\}_{S \subset N},\left(\succ_{i}\right)_{i \in N}\right)$ is the stochastic cooperative game with n players, and the stochastic payment $\left(d^{N}, r^{N}\right) \in Z(N)$. And if $i, j \in N$ exists, and satisfies

$\left\{\begin{array}{c}s_{i j}(d, r)>s_{j i}(d, r) \\ \left(d^{N}, r^{N}\right)_{j}>\left(\tilde{d}^{\{j\}}, \tilde{r}^{\{j\}}\right)\end{array}\right.$

, so at the position of $\left(d^{N}, r^{N}\right)$, i outweights j.

Definition 2.5: At the position of $\left(d^{N}, r^{N}\right)$, if i doesn't outweight $\mathrm{j}$, and $\mathrm{j}$ doesn't outweight $\mathrm{i}$, so $\mathrm{i}$ and $\mathrm{j}$ are equilibrium at the position of $\left(d^{N}, r^{N}\right)$, i.e. 
$\left\{\begin{array}{l}\left(s_{i j}(d, r)-s_{j i}(d, r)\right)\left(\left(d^{N}, r^{N}\right)_{j}-\left(\tilde{d}^{\{j\}}, \tilde{r}^{\{j\}}\right)\right) \leq 0 \\ \left(s_{j i}(d, r)-s_{i j}(d, r)\right)\left(\left(d^{N}, r^{N}\right)_{j}-\left(\tilde{d}^{\{j\}}, \tilde{r}^{\{j\}}\right)\right) \leq 0\end{array}\right.$

Definition 2.6: The kernel $K(\Gamma)$ of the stochastic cooperative game is the collectivity of all these distributions, and for the distribution $\left(d^{N}, r^{N}\right)$, any two players are in equilibrium, i.e.

$$
K(\Gamma)=\left\{\left(d^{N}, r^{N}\right) \in Z(N) \mid \begin{array}{l}
\left(s_{i j}(d, r)-s_{j i}(d, r)\right)\left(\left(d^{N}, r^{N}\right)_{j}-\left(\tilde{d}^{\{j\}}, \tilde{r}^{\{j\}}\right)\right) \leq 0 \\
\forall i, j \in N, i \neq j
\end{array}\right\}
$$

Definition 2.7: The nucleolus $N u(\Gamma)$ of the stochastic cooperative game is the collectivity of those distributions which minimize $Q(d, r)$ according to the dictionary sequence, i.e.

$$
N u(\Gamma)=\left\{(d, r) \in Z(N) \mid Q(d, r) \leq_{L} Q(\tilde{d}, \tilde{r}), \forall(\tilde{d}, \tilde{r}) \in Z(N)\right\} .
$$

\section{Main conclusions}

Theorem 3.1: Suppose $\Gamma$ is the stochastic cooperative game, so

$$
N u(\Gamma) \subseteq K(\Gamma)
$$

Prove: Suppose $N u(\Gamma)=\{(d, r)\}$, prove $(d, r) \in K(\Gamma)$ by the proof method of contradiction.

If $(d, r) \notin K(\Gamma)$, so $i, j \in N, i \neq j$ exists to make

$s_{i j}(d, r)>s_{j i}(d, r)$

$$
\left(d^{N}, r^{N}\right)_{j}>\left(\tilde{d}^{\{j\}}, \tilde{r}^{\{j\}}\right) \quad(\tilde{d}, \tilde{r}) \in Z(\{i\})
$$

Take

$0<\delta<\left\{\frac{1}{2}\left(s_{i j}(d, r)-s_{j i}(d, r)\right),\left(d^{N}, r^{N}\right)_{j}-\left(\tilde{d}^{\{j\}}, \tilde{r}^{\{j\}}\right)\right\}$

And $(\bar{d}, \bar{r})$

$$
(\bar{d}, \bar{r})_{k}=\left\{\begin{array}{cc}
\left(d^{N}, r^{N}\right)_{i}+\delta, \quad k=i \\
\left(d^{N}, r^{N}\right)_{j}-\delta, \quad k=j \\
\left(d^{N}, r^{N}\right)_{k}, \quad k \neq i, j
\end{array}\right.
$$

, so $(\bar{d}, \bar{r}) \in I R(N)$. Rank $Q(d, r)$ and $Q(\bar{d}, \bar{r})$ according to the dictionary sequence, and because $\forall S \subseteq N$, so

(1) When $S \subseteq M_{i j}, e[S,(\bar{d}, \bar{r})]=e[S,(d, r)]-\delta$

(2) When $S \subseteq M_{j i}, e[S,(\bar{d}, \bar{r})]=e[S,(d, r)]+\delta$

(3) When $S \notin M_{i j} \cup M_{j i}, e[S,(\bar{d}, \bar{r})]=e[S,(d, r)]$

So, different components of $Q(d, r)$ and $Q(\bar{d}, \bar{r})$ certainly are the excess values in $M_{i j}$ or $M_{j i}$. And because $M_{i j}(d, r)>M_{j i}(d, r)$, and 


$$
\begin{aligned}
s_{i j}(\bar{d}, \bar{r}) & =\max _{S \in M_{i j}} e[S,(d, r)]-\delta=s_{i j}(d, r)-\delta> \\
& S_{j i}(d, r)+\delta= \\
& \max _{S \in M_{j i}} e[S,(d, r)]+\delta= \\
& \max _{S \in M_{j i}} e[S,(\bar{d}, \bar{r})]=s_{j i}(\bar{d}, \bar{r})
\end{aligned}
$$

Therefore, when comparing $Q(d, r)$ with $Q(\bar{d}, \bar{r})$, the different maximum components of $Q(d, r)$ and $Q(\bar{d}, \bar{r})$ certainly are the excess values in $M_{i j}$, and

$$
\begin{gathered}
s_{i j}(\bar{d}, \bar{r})=e\left[S_{0},(\bar{d}, \bar{r})\right]= \\
e\left[S_{0},(d, r)\right]-\delta= \\
s_{i j}(d, r)-\delta< \\
s_{i j}(d, r)
\end{gathered}
$$

So, $Q(\bar{d}, \bar{r}) \leq_{L} Q(d, r)$.

It is contrary with $N u(\Gamma)=\{(d, r)\}$, so $N u(\Gamma) \subseteq K(\Gamma)$. End.

Theorem 3.2: The nucleolus of the stochastic cooperative game is the subset of the intersection set of the kernel and the minimum core, i.e.

$N u(\Gamma) \subseteq K(\Gamma) \bigcap L C(\Gamma)$.

Prove: Suppose $(d, r) \in N u(\Gamma)$, and $(\tilde{d}, \tilde{r}) \in C_{\varepsilon}(\Gamma)$, for any one alliance $\mathrm{S}$,

$e[S,(\tilde{d}, \tilde{r})] \leq \varepsilon$

So, $\max _{S \subseteq N} e[S,(d, r)] \leq Q_{1}(d, r) \leq Q_{1}(\tilde{d}, \tilde{r}) \leq \max _{S \subseteq N}[S,(\tilde{d}, \tilde{r})] \leq \varepsilon$

, and $(d, r) \in C_{\varepsilon}(\Gamma)$. From the definition 2.5, $N u(\Gamma) \subseteq K(\Gamma)$, so $(d, r) \in K(\Gamma)$, and

$(d, r) \in C_{\varepsilon}(\Gamma) \cap K(\Gamma)$

i.e. $N u(\Gamma) \subseteq K(\Gamma) \bigcap L C(\Gamma)$

, end.

Theorem 3.3: Suppose $\Gamma$ is the stochastic cooperative game, so when $(d, r) \in K(\Gamma)$ and only when $(d, r) \in Z(N)$,

$s_{i j}(d, r)=s_{j i}(d, r), \quad \forall i, j \in N, i \neq j$

Prove: From the super-additivity of $\Gamma$, for $\forall S \subseteq N, \forall T \subseteq N$,

$d_{i}^{S \cup T}+r_{i}^{S \cup T} X_{S \cup T} \geq_{i} d_{i}^{S}+r_{i}^{S} X_{S} \quad(\forall i \in S)$

$d_{i}^{S \cup T}+r_{i}^{S \cup T} X_{S \cup T} \geq_{i} d_{i}^{T}+r_{i}^{T} X_{T} \quad(\forall i \in T)$

First, from the definition of $K(\Gamma),(d, r) \in I R(N)$ satisfying the formula (2-5) certainly belongs to $K(\Gamma)$. So only need to prove $\forall(d, r) \in K(\Gamma)$, and $(d, r)$ certainly satisfies the formula (2-6) and the formula (2-7). Note

$\alpha=\max \left\{s_{i j}(d, r) \mid i, j \in N, i \neq j\right\}$ 


$$
\begin{aligned}
& \Omega=\{S \mid S \neq \varnothing, S \neq N, e[S,(d, r)]=\alpha\} \\
& M=\bigcap_{S \in \Omega} S
\end{aligned}
$$

So, $M \neq N$.

Prove $M=\varnothing$, and if it is not true, suppose $k \in M$, take any $l \in N \backslash M$, from the definition of M, $S_{0} \in \Omega$ exists to make $S_{0} \in M_{k l}$, and

$\Omega \cap M_{k l}=\varnothing$

So,

$s_{k l}(d, r)=e\left[S_{0},(d, r)\right]=\alpha>e[S,(d, r)], \forall S \in M_{l k}$

, and

$s_{k l}(d, r)>s_{l k}(d, r)$

From $(d, r) \in K(\Gamma),(d, r)_{l}=\left(d^{\{l\}}, r^{\{l\}}\right)$ and $(\forall l \in N \backslash M)$. So from the formula (2-6) and the formula (2-7),

$$
\begin{aligned}
& \alpha=e\left[S_{0},(d, r)\right]= \\
& \quad \sum_{i \in S_{0}}\left(\tilde{d}^{S_{0}}, \tilde{r}^{S_{0}}\right)_{i}-\sum_{i \in S_{0}}\left(d^{N}, r^{N}\right)_{i}= \\
& \quad \sum_{i \in S_{0}}\left(\tilde{d}^{S_{0}}, \tilde{r}^{S_{0}}\right)_{i}-\sum_{i \in N}\left(d^{N}, r^{N}\right)_{i}+\sum_{i \in N \backslash S_{0}}\left(d^{\{i\}}, r^{\{i\}}\right)_{i} \leq \\
& X_{N}-\sum_{i \in N}\left(d^{N}, r^{N}\right)_{i}=0 \\
& \left(\tilde{d}^{\{l\}}, \tilde{r}^{\{l\}}\right) \in Z(\{l\}) \text { exists to make } \\
& e[\{l\},(d, r)]=\sum_{i \in\{l\}}\left(\tilde{d}^{\{l\}}, \tilde{r}^{\{l\}}\right)_{i}-\sum_{i \in\{l\}}\left(d^{N}, r^{N}\right)_{i}=0 \geq \alpha
\end{aligned}
$$

, so $\{l\} \in \Omega$.

It is contrary with $\Omega \bigcap M_{k l}=\varnothing$. So $M=\varnothing$.

Second, prove $(d, r)$ satisfies the formula (2-5). If $i, j \in N$ exists to make

$s_{i j}(d, r)>s_{j i}(d, r)$

, so $\left(d^{N}, r^{N}\right)_{l}=\left(\tilde{d}^{\{l\}}, \tilde{r}^{\{l\}}\right)$.

From $M=\varnothing, \mathrm{S} 1$ without $\mathrm{i}$ in $\Omega$ certainly exists, and according to the formula (2-5) and the formula (2-6),

$$
\begin{aligned}
e\left[S_{1} \cup\{l\},(d, r)\right]= & \sum_{i \in S_{1} \cup\{l\}}\left(\tilde{d}^{S_{1} \cup\{l\}}, \tilde{r}^{S_{1} \cup\{l\}}\right)_{i}-\sum_{i \in S_{1}}\left(d^{N}, r^{N}\right)_{i}-\left(\tilde{d}^{\{j\}}, \tilde{r}^{\{j\}}\right)_{i} \geq \\
& \sum_{i \in S_{1}}\left(\tilde{d}^{S_{1}}, \tilde{r}^{S_{1}}\right)_{i}-\sum_{i \in S_{1}}\left(d^{N}, r^{N}\right)_{i}= \\
& e\left[S_{1},(d, r)\right]
\end{aligned}
$$

, so $s_{j i}(d, r) \geq e\left[S_{1} \cup\{j\},(d, r)\right] \geq e\left[S_{1},(d, r)\right]=\alpha \geq s_{i j}(d, r)$.

It is contrary with the formula (2-8). End.

\section{References}

Gao, Zuofeng, \& Bai, Hongxin. (2008). The $\varepsilon$-Core of a n-person Stochastic Cooperative Game. Modern Applied Science. No.2(2). P.43-46.

Gao, Zuofeng, Wang, You \& Wang, Guocheng. (2006). Countermeasure Theory and Economic Management 
Decision-making. Beijing: China Forestry Press.

Suijs J, Borm P, De Waegenaere A, et al. (1999). Cooperative game with stochastic payoffs. European Journal of Operational Research. No.113. P.193-205.

Suijs J \& Borm P. (1999). Stochastic cooperative game: superadditivity, convexity, and certainty equivalents. Game and Economic Behavior. No.27. P.331-345.

Suijs J, De Waegenaere A \& Borm P. (1998). Stochastic cooperative game in insurance. Insurance: Mathematics \& Economics. No.22. P.209-228.

Xie, Zheng. (2004). Theory of Countermeasure. Changsha: National University of Defense Technology Press. P.156-180.

Zuofeng Gao, Hongxin Bai \& Suting Zhang. (2008). The $\varepsilon$-core of ${ }^{n}$-person Stochastic Cooperative Game. Modern Applied Science. No.2(2). P.71-73. 